\title{
Implementation of the trauma registry as a tool for quality improvement in trauma care in a brazilian hospital: the first 12 months
}

\section{Implantação de registro de trauma como ferramenta para melhorar a qualidade do atendimento a traumatizados: os primeiros 12 meses}

José Gustavo Parreira, TCBC-SP 1; Tércio de Campos, tCBC-SP2; Jacqueline A. Gianinni Perlingeiro,TCBC-SP1; Silvia C. Solda, TCBC-SP1; José Cesar Assef, TCBC-SP1; Augusto Canton Gonçalves 3 ; Bruno Malteze Zuffo ${ }^{3}$; Calo Gomes Floriano 3 ; Erik Haruk de Oliveira3; Renato Vieira Rodrigues de Oliveira ${ }^{3}$; Amanda lima Oliveira3; Caio Gullo de Melo ${ }^{3}$; Cristiano Below3; Dino R. Pérez Miranda ${ }^{3}$; Gabriella Colasuonno Santos ${ }^{3}$; Gabriele Madeira de Almeida ${ }^{3}$; Isabela Campos Brianti ${ }^{3}$; Karina Baruel de Camargo Votto ${ }^{3}$; Patrick Alexander Sauer Schues ${ }^{3}$; Rafael Gomes dos Santos ${ }^{3}$; Sérgio Mazzola Poli de Figueredo3; Tatiani Gonçalves de Araujo3; Bruna do Nascimento Santos3; Laura Cardoso Manduca Ferreira3; Giuliana Olivi Tanaka3; Thiara Matos ${ }^{3}$; Maria Daiana da Sousa ${ }^{4}$; Samara de Souza Augusto ${ }^{4}$

\section{A B S S T R A C T}

\begin{abstract}
Objective: to analyze the implementation of a trauma registry in a university teaching hospital delivering care under the unified health system (SUS), and its ability to identify points for improvement in the quality of care provided. Methods: the data collection group comprised students from medicine and nursing courses who were holders of FAPESP scholarships (technical training 1) or otherwise, overseen by the coordinators of the project. The itreg (ECO Sistemas-RJ/SBAIT) software was used as the database tool. Several quality "filters" were proposed to select those cases for review in the quality control process. Results: data for 1344 trauma patients were input to the itreg database between March and November 2014. Around $87.0 \%$ of cases were blunt trauma patients, $59.6 \%$ had RTS $>7.0$ and $67 \%$ ISS $<9$. Full records were available for 292 cases, which were selected for review in the quality program. The auditing filters most frequently registered were laparotomy four hours after admission and drainage of acute subdural hematomas four hours after admission. Several points for improvement were flagged, such as control of overtriage of patients, the need to reduce the number of negative imaging exams, the development of protocols for achieving central venous access, and management of major TBI. Conclusion: the trauma registry provides a clear picture of the points to be improved in trauma patient care, however, there are specific peculiarities for implementing this tool in the Brazilian milieu.
\end{abstract}

Key word: Multiple Trauma. Wounds and Injuries. Trauma Severity Indices. Medical Records. Quality Control.

\section{INTRODUCTION}

lnoting 2013, 151,683 trauma patients died in Brazil, amounting to a rate of approximately 415 deaths per day or 17 per hour ${ }^{1}$. There was a $21 \%$ increase in mortality due to external causes between 2003 and 2013, accounting for $12.9 \%$ of all deaths registered in Brazil ${ }^{1}$. This represents the third most frequent cause of death among Brazilians and, in the population aged 5-39 years, is the leading cause of death in Brazil.Given that trauma predominantly affects younger individuals, it is a cause which most consumes years of productive life.Based on these figures, it can be inferred that trauma is a major burden of society².
On the $8^{\text {th }}$ of July 2013, the Ministry of Health approved the project "Line of Attention to Trauma", which lays out clear objectives to change this scenario ${ }^{3}$. Under the project, several measures are proposed to improve care, including the implementation of quality control programs in trauma patient care (PQAT), defined as methods and processes created to continuously monitor the diagnosis, management and evolution of trauma patients ${ }^{4,5}$. The PQAT avails of several different tools, such as trauma registries (TR). morbimortality meetings, investigation of avoidable deaths, development of filters for auditing, morbimortality review committees, cycle outcomes and quality process metrics, among others ${ }^{5-9}$

1. Disciplina de Cirurgia de Urgência. Departamento de Cirurgia. Faculdade de Ciências Médicas da Santa Casa de São Paulo; 2. Departamento de Cirurgia. Faculdade de Ciências Médicas da Santa Casa de São Paulo; 3. Curso de Medicina da Faculdade de Ciências Médicas da Santa Casa de São Paulo; 4. Curso de Enfermagem da Faculdade de Ciências Médicas da Santa Casa de São Paulo. 
The TR is a database which holds specific characteristics of trauma cases, including demographic and epidemiological data, trauma mechanism, pre-hospital care information, diagnostic procedures, treatment administered, patient evolution and costs ${ }^{5,9-11}$. These registries must store information on traumatic injuries, classify them, define their severity and group the information for individual and collective analyses.

The study hypothesis is that the Trauma Registry (TR) is a useful and cost effective tool for identifying specific points to be addressed in a patient trauma care quality improvement process. However, implementation of this system within a hospital that predominantly treats users from the Unified Health System (SUS) poses certain challenges, particularly related to the absence of a local culture for data collection. Few hospitals in Brazil have this experience.

The objective of this study was to analyse the process of implementing a Trauma Registry in a university teaching hospital delivering care predominantly to SUS users, and its ability to identify issues and their impact on improving quality of the process.

\section{METHODS}

The study was submitted to the Research Ethics Committee of the Irmandade da Santa Casa de São Paulo Hospital and approved under decision number 656.666.

The software used for the collection and storage of data was itreg, developed by ECO - Empresa de Consultoria e Organização em Sistemas e Editoração Ltda., in conjunction with the Brazilian Society of Integrated Care for the Trauma Patient (SBAIT). The itreg uses the internet and a site only accessible by authorized users holding the password. Besides the data entry screens, there are preestablished dashboards which allow a clear assessment of the most important points for assessing the quality of trauma patient care in real time.

Briefly, the itreg stores data pertaining to patient identification, trauma mechanism, pre-hospital care, transport, initial care, complementary exams (laboratory and imaging), injuries diagnosed, severity of sample (trauma scales), treatment administered, complications, hospital discharge, quality filters (national and international) and $I C D$, among others. Stratification of severity is performed based on calculation of the following scales: Glasgow coma scale (GCS) ${ }^{12}$, Revised Trauma Score (RTS) ${ }^{13}$, Abbreviated Injury Scale (AIS-1990) ${ }^{14}$, Injury Severity Score (ISS) ${ }^{15}$, NISS, NTRISS ${ }^{16}$ TRISS $^{17}$

Data collection was carried out by the Trauma Study Group (GET), formed by students from medicine and nursing courses of the School of Medical Sciences of Santa Casa de São Paulo. First, a ten hours theoretical-practical training course on data collection for trauma patients was given. A pilot study was then conducted in which data were collected for all trauma patients admitted to the emergency room of the Central Emergency Service of the Irmandade da Santa Casa de Misericórdia de São Paulo Hospital over a period spanning three months, commencing in March 2014. After assessing the quality of the data and refining the process, definitive data collection was started for an initial period of 12 months.

During this period, the Quality in Trauma Patient Care Project (PQAT) was implemented within the Emergency Service of the ISCMSP, based on the information from the Trauma Registry (TR).First, meetings were held involving all the attending physicians from the surgical team of the emergency service to present the project and take suggestions to be incorporated into the program. Meetings were also held with the resident doctors from the Surgical Department, showing how the program operates. After these preliminary definitions, the PQAT was officially deployed and run using the following tools: Routine discussion meetings (RD) - These meetings were held monthly on the $2^{\text {nd }}$ Tuesday of each month, with discussions centered mainly on routines for patient care. Those wishing to provide input were also invited to take part. A number of matters were tabled and included in an agenda for future discussions; Morbidity and mortality meetings (MM) - MM meetings were split into two parts.TR reports are presented first, along with the key numbers and indicators. The next topic is the review of cases pre-selected based on the quality filters of the TR. Auditing filters are variables which are more frequently associated with breaches of protocol or failed care. This calls for thorough case by case review, in a bid to identify the underlying circumstances that led to these occurrences. This process is carried out by an independent auditor (not involved with care of the patient), who reports at the morbidity and mortality meetings of the group. The case reporter makes pertinent comments at this time. Based on this discussion, the points to be addressed by the PQAT are identified. This meeting is confidential in order to protect the parties involved in the discussion of the clinical case. PQAT Reports - Based on the ideas raised at RD and MM meetings, a report is produced, which is reviewed by the Director of the Emergency Service and by the other participants.Following approval, this report is sent to those interested (attending physicians, resident doctors, nurses, managers, etc.) via the internet as required. Upon conclusion of the project, the group intends to produce a manual containing all the reports discussed in order to assist in the PQAT of other interested institutions. Continued education - Drawing on the points discussed at the previous meetings, an issue is chosen for weekly review. As decided by the attending doctors, an article is sent by email to all participants for review of the subject and updating of the team. Depending on the topic, a specific meeting to define the protocol is decided and scheduled.

In the present study, the process of implementing the TR as a whole was analyzed, including the obstacles 
encountered and solutions found. An assessment of the data collected was carried out in an effort to identify key points on which trauma patient care can be improved by specific interventions and training of the group. The preliminary phase of the PQAT was also evaluated, specifically the problems observed and the proposed solutions to remedy them.

\section{RESULTS}

\section{Data collected}

As of 24/11/2014, data for 1344 trauma patients had been input to itreg. Around $87 \%$ were blunt trauma patients, among which the most common mechanisms were falls from the same level (17.3\%), followed by falls from height (16.1\%), motorcyclists (15.6\%) and being struck by a vehicle (15.2\%).

Analysis of sample severity revealed that the vast majority of patients (89.1\%), at admission, had a Glasgow coma scale score of $13-15$ while only $7.6 \%$ had systolic arterial pressure under $100 \mathrm{mmHg}$. The sample was characterized by the large number of patients (59.6\%) with RTS $>7.0$ (without significant physiological changes) and ISS $<9$ (67\%) (without serious injuries) (Figure 1).

From these records, 292 were reviewed and completed, forming the sample for the assessment of the quality control variables. Figure 2 shows patient destination following assessment. The majority of those admitted remained within the emergency service, either in a ward bed, emergency room bed or semi-intensive care unit. Only 21 out of the 292 closed files were admitted directly $(<24$ hours) to intensive care. Among patients with closed files, the most common treatments administered were thoracic drainage $(12 \%)$, laparotomies $(6.8 \%)$ and conservative management of limb fractures (5.8\%).

The most frequent complications were respiratory failure and hemodynamic shock.latrogenic pneumothorax was noted in seven out of the 292 patients with closed files (Figure 3). Twenty-seven patients died, representing 9.2\% of closed files. The most common causes of death were traumatic brain injury (11) and hemorrhage (5).

The auditing filters most frequently identified were laparotomy four hours after admission (3.4\%), commencement of nonoperative management for injuries to the liver, spleen, kidney and/or pancreas (2\%), and drainage of acute subdural hematomas four hours after admission (1.7\%) (Figure 4). Lethality was highest for RTS scores $<2$ and for ISS scores $>25$ (Figure 5).

\section{DISCUSSION}

There is no question that TR yield valuable information $4,5,18$. In the context of quality control and trauma care, the outcome and the costswould be more

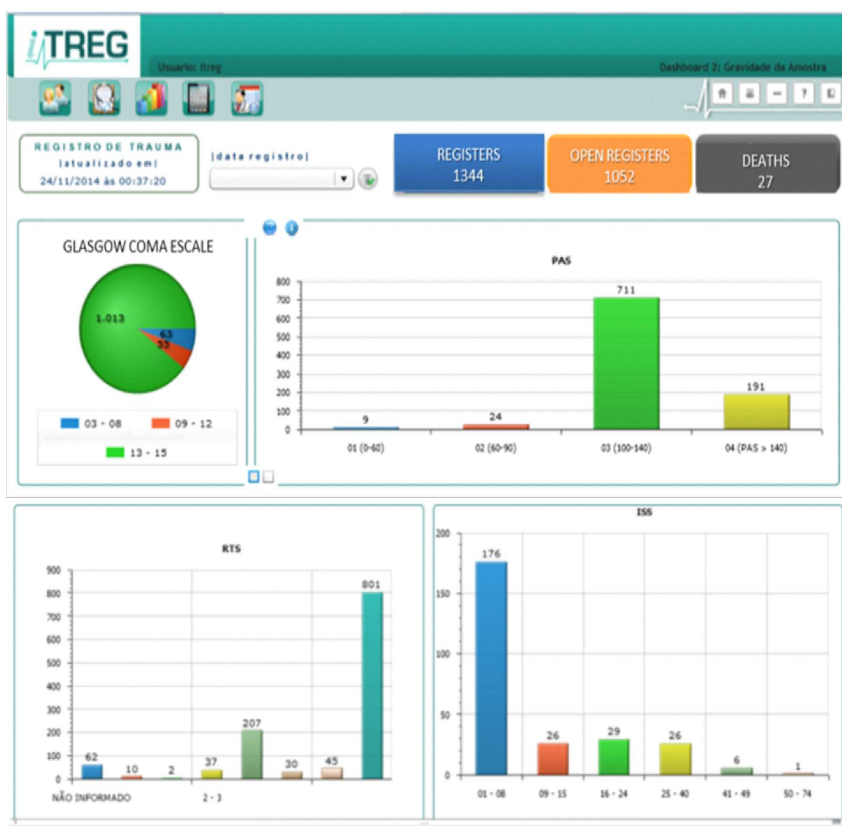

Figure 1 - Severity of the sample.

Source: Emergency Service of the Irmandade da Santa Casa de Misericórdia de São Paulo Hospital.

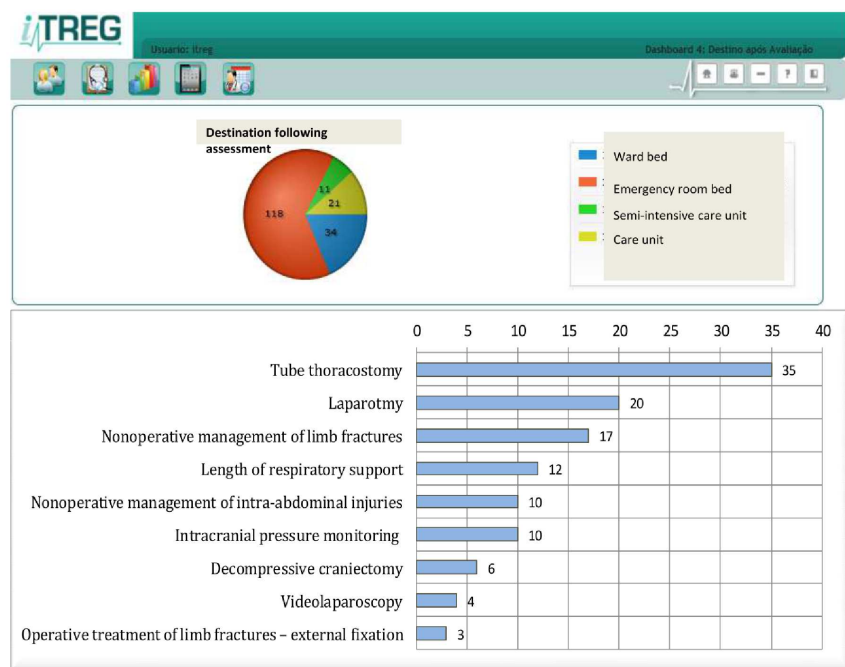

Figure 2 - Destination after initial assessment and most frequently administered treatment options in 292 patients with closed files.

Source: Emergency Service of the Irmandade da Santa Casa de Misericórdia de São Paulo Hospital.

accurately assessed. The main challenge might be implementing the TR, particularly in settings without a culture of data collection and quality control processes ${ }^{19-22}$

\section{Analysis of TR implementation}

The pilot project brought many obstacles to light. The first of these was identifying and locating trauma patient amid the flow of patients through the emergency room, 


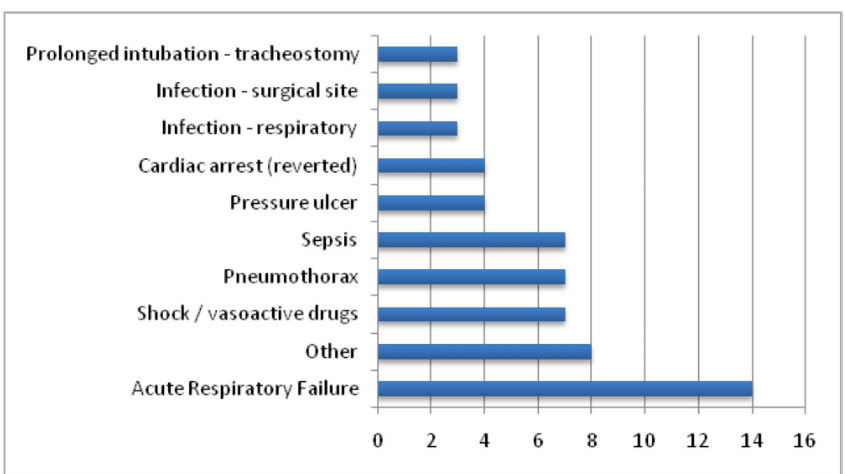

Figure 3 - Most frequent complications observed in 292 patients with closed files.

Source: Emergency Service of the Irmandade da Santa Casa de Misericórdia de São Paulo Hospital.

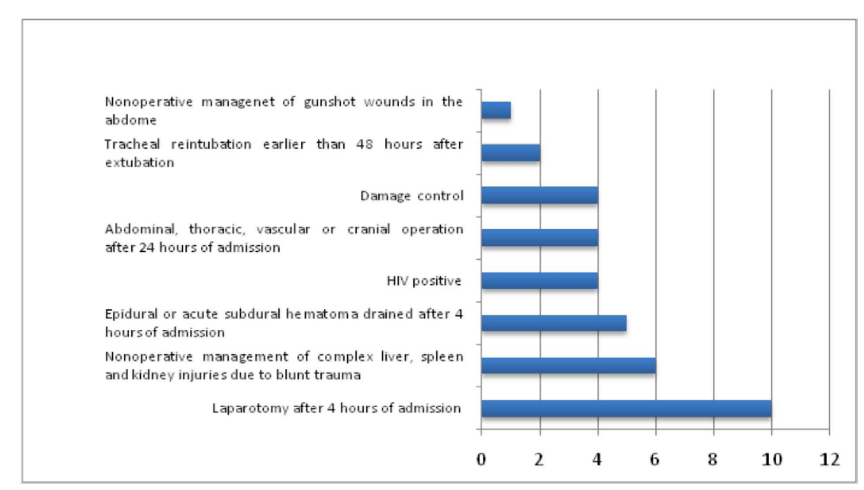

Figure 4 - $\quad$ Auditing filters for trauma observed in 292 patients with closed files.

Source: Emergency Service of the Irmandade da Santa Casa de Misericórdia de São Paulo Hospital.

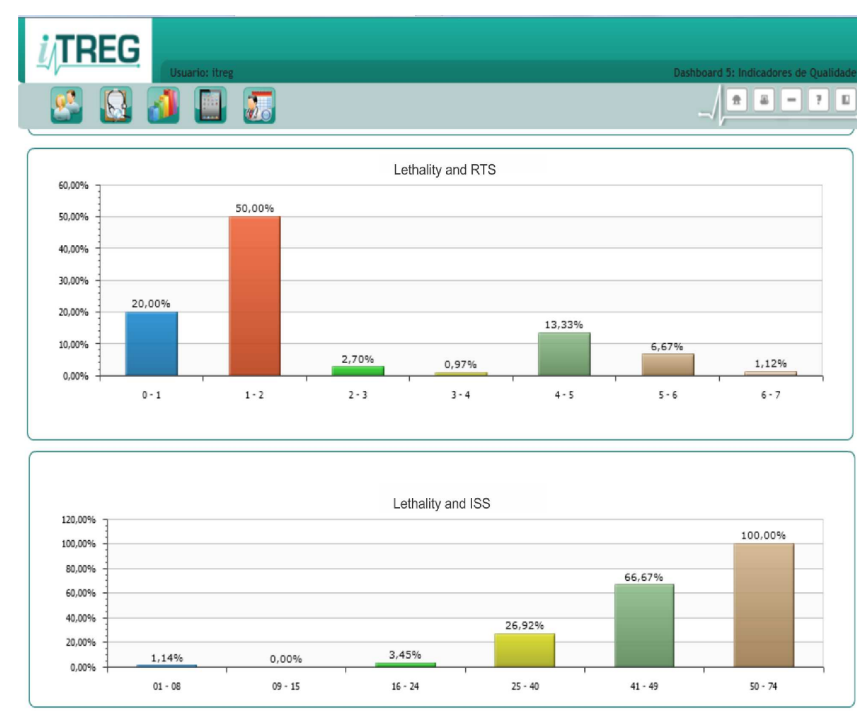

Figure 5 - Distribution of lethality (within 30 days) according to RTS and ISS severity scales.

Source: Emergency Service of the Irmandade da Santa Casa de Misericórdia de São Paulo Hospital. whose occupancy averages 100-120 clinical and surgical cases (despite having "only" 62 beds).

The entry point of trauma patients is via the Emergency Room at "Trauma Room 1". Depending on the case, the patient may be referred to undergo complementary exams, or transferred to the ward, intensive care and/or surgical center. Since there are often no beds available initially, patients may remain on gurneys for a period. The space within the PSC where patients are placed temporarily varies according to demand and availability, and changes throughout the day, precluding attempts to list the patients. Given that the service handles an average of 20-30 trauma patients daily, besides other PSC patients, locating a particular patient amidst this volume became problematic.

This was exacerbated by the fact that only one GET member was present per shift (12-hour night shift) where covering all these locations at once proved impossible. The members of the GET were often not in the trauma room at the time of patient arrival. The most obvious alternative was to ask administrative clerks and or resident doctors to advise GET members upon entry of a trauma patient, but this often did not take place, compromising admissions data collection.

In an effort to complete the necessary information, data was drawn from patient medical charts. One of the major points was identified during the initial phase: the information needed for quality control of trauma care was missing from medical charts. This relates mainly to data on respiratory rate and Glasgow coma scale, needed to calculate the trauma measures and probability of survival. In some cases, the description of the clinical decision-making process in the charts was unclear, which hampered the GET team members who had problems completing the TR.

The situations above led to another problem: some files were "opened" by scholarship holders but not closed (i.e. not completed with all the necessary data) prior to patient discharge from the hospital. This resulted in a very large number of "open" files, which contained only information collected at admission plus exams ordered, yet not including exam results, injuries diagnosed and treatment proposed nor details of complications and deaths. Without this data, setting up a quality program would not be possible. The itreg revealed this problem, showing a large number of open files together with a low rate of injuries and complications.

The existence of a learning curve for data collection was clear. The GET comprised medical students in their second or third yearand nursing students in their fourth year of the course. All had an evident learning curve. During the pilot project period, numerous basic doubts were brought up at the meetings and all learned from the experience. The main limitations were difficulty understanding the written annotations in the medical charts, treatment procedures, clinical outcomes and grading 
anatomical injuries using the AIS-90 table.

These issues were discussed at the GET meetings and those of PQAT members. The primary objective was to set-up an information system to alert the GET upon entry of a trauma patient to the trauma room, and to facilitate the process of finding the trauma patients still in the central emergency room and those who had been transferred to other units of the hospital. To this end, the following steps were taken: a) The residents responsible for attending the trauma patients were made aware that if no GET member was present upon admission of a trauma patient then a member should be alerted; b) A group was set-up on the WhatsApp application called Trauma alert. The most qualified residents on the shift sent a daily alert to GET members detailing the trauma patients undergoing followup and/or operated in the last 12 hours, along with their diagnosis, registration and procedures performed; c) A table of patients admitted to hospital and followed by the GET was created and made available on the "cloud" using the spreadsheet application and updated daily by GET members. This listing served less to locate the patient bed but more to inform whether the patient had left the hospital; d) A log book registering all trauma patients included in the itreg during each shift was introduced. A photograph of the log book page was sent daily by the shift leader to the WhatsApp group GET it and served as a control for patients input into the program; e) GET members were tasked with starting their shift by doing a "round" of all the trauma patients present in the PSC, done together with the group of residents and assistants taking over the shift, thereby guiding objectives; f) A subgroup of the GET responsible for the daily visit to the hospitalized patients was set-up. This subgroup was called "horizontal" as its work times were spread over the five working days of the week to allow linear follow up. Because they had the afternoons free, nurses and nursing students were chosen for this task. This was an important point, since it established a "trauma visit" which involved not only the scholarship holders but also the group researchers.

Another solution found to help optimize the closure of files was to schedule "closure meetings", held weekly at a dedicated time outside shifts or other commitments. Based on the information drawn from medical charts, the data on the itreg was reviewed and incomplete fields filled out up to the time of patient exit. The presence of a more experienced researcher was found to be fundamental to ensure the information entered into itreg was truly reliable. Interpreting the medical chart is often challenging for students, calling for more clinical experience.

Despite all these resources, it was noted that gaps existed in the shift roster, reducing the number of patients input into itreg. Since there were a number of scholarships unused because they were different types, a request for reallocation of the available funds to TT1 type scholarships was made. A further 15 TT1 scholarships were approved and another selection process began. Since then, the GET has scholarship holders carrying out data collection during the night shift (12 hours) seven days a week, while coverage is 24 hours at weekends and there is also the afternoon group (horizontal group) during week days.

In Brazil, the vast majority of trauma patients are first attended in hospitals under the SUS.The financial difficulties faced by some of these institutions are well known. Most of the hospitals cannot afford to hire (through public admissions procedures) specialized personnel solely to collect data. Resources are generally allocated to other areas, particularly in healthcare delivery.

Nevertheless, there are numerous benefits of quality control in trauma care, the "heart" of which is the information collected and stored in the TR. It is important to grasp the concept that quality control is not a means to punish people but a tool for protecting them. Once the initial obstacles have been overcome, the analysis of the data becomes the main tool for improving the service. We believe that when results improve, the rate of complications will fall, also promoting shorter hospital stays, lower costs with intensive therapy, nutritional support and antimicrobials, among a series of other advantages.

Many challenges were faced during the implementation of the project as a whole. We provided a detailed description of the most important points in order to help pave the way for other researchers and hospital managers who choose to pursue this path. These obstacles go far beyond mere tailoring of the software for use in Brazil, but also involve the training of an inexperienced group to carry out a relatively complex procedure. Once all the elements have been put in place, the challenge is to keep the group together and working smoothly, since many individuals are having to work together as a team, giving rise to specific problems.

The TR originated in the developed trauma systems from North America, which spread quickly to Europe. The culture of quality control is directly linked to improvements in performance on many fronts, including financial.Centers that fail to submit their data to regulatory agencies do not receive funding. This does not occur in Brazil, which may be one of the reasons explaining the low number of services with active TR.

There is a major difference between the model proposed in the present study and that observed in centers with TR experience. At more developed trauma centers, data collection is done professionally. There is the role of Trauma Nurse, with specific training and paid solely for working on $\mathrm{TR}^{7}$. This professional has access to the medical charts containing information on treatment and feeds this data into the TR retrospectively. The present project also differs in another respect. If this professional model had been implemented instead, invaluable information on prehospital care and admission would have been lost, since this data often goes unrecorded in medical charts. One of the insights gleaned from implementing this program in the hospital was the need to create tools to improve the 
filling out of information in medical charts. Until this need is addressed, a system of "prospective collection" has been introduced, requiring the efforts of a large group of people.

Regarding the data collected, there is much to be done to achieve complete analysis. Ideally, all the open records should be "closed" when the patient leaves the hospital. This proved impossible under the current conditions, generating information which does not lend itself to scientific interpretation. Current efforts are focused on attempting to reduce the number of open files through the closure meetings outlined earlier.

However, a number of key points have been identified in this initial assessment. A large number of trauma patients treated by the service were found to have no major injuries. Only a small percentage of patients presented serious injuries and remained hospitalized after initial assessment. This phenomenon is known as "overtriage" and occurs when mild cases are referred to advanced trauma centers (tertiary, quaternary and/or university teaching hospitals), when they could be treated in secondary hospitals. The consequences are clear and exacerbated in an emergency service with a high volume of patients such as ours: patients, even devoid of serious injuries, require professionals for care, and also for ordering and scheduling complementary exams. This diverts some of the team to a group of trauma patients without major injuries, whilst other more serious cases need their assistance; gurneys and physical space are occupied in the emergency service until all the teams have gone over the patient and exam results again; there is high demand for use of imaging methods, such as X-ray, ultrasound and tomography. The patient flow of the service is negatively impacted by a large number of exams testing negative.

The occurrence of a high rate of negative exams may be due to several factors:the cause of this phenomenon may be related to failure to follow protocols for ordering exams, which may indicate the need for targeted actions in the quality program for group training; the protocols for ordering exams are flawed and should be revised. This is because some protocols allow a high rate of negative exams.In trauma, many severe injuries can be present in asymptomatic patients and an active search can lead to negative exams; these exams overload the group of professionals that attend trauma patients and also the equipment available, causing the problems cited above.

The most frequent complications observed were systemic in nature: respiratory failure and shock requiring vasoactive drugs. Local complications were less common. These complications are likely related to the systemic inflammatory response secondary to trauma (first hit) or to an infectious insult (second hit). Other important points for deployment in the quality program were the presence of iatrogenic pneumothorax and pressure ulcers. Both of these complications can be addressed by the application of protocols to reduce their occurrence through education and preventive measures. As observed in most casuistics, traumatic brain injury was the most common cause of death, followed by hemorrhaging. Likewise, specific protocols can be developed and applied to target these problems.

The evaluation of the quality filters in the TR revealed that some of these situations were clearly more frequent, such as subdural hematoma drainage four hours after admission. Each of these cases was reviewed to identify the issues that contributed to delays in surgical procedures or to ascertain whether failures in the process took place. Employing the TR for its primary purpose, namely, to identify key points where the quality program can be most effective, produced important results. One of the most effective ways of improving quality was the identification of cases for review at the closed MM meetings. The TR was pivotal in flagging those cases associated with points to be improved in the process. This appeared to be one of the greatest benefits of implementing the TR.

Concerted efforts to maintain the quality of care delivered to trauma patients have been made for years in the institution. The PQAT served to organize and standardize this process. The engagement of the group of professionals hired is variable and hinges wholly on personal motivation. However, many physicians hired to practice in emergency services have time available only for healthcare assistance. Extra meetings and continued education were not envisaged, which can sometimes pose a problem. Attendance at routine discussion and $\mathrm{MM}$ meetings depends on this variable. In order to raise the knowledge level of the group on the PQAT, reports were made available to the attending physicians of the service by email. We feel that, within a mature quality program, all professionals involved in trauma patient care should have a remunerated dedicated period every week to participate in the PQAT, which also includes continued education.

Another positive aspect observed at the meetings for defining routines is the involvement of various professionals including those from different medical specialties. This forms a task force which, working together, is better able to resolve problems and pursue action on different fronts.For example, at a meeting to define transfusion protocols, there was involvement from not only the general surgeon but also blood bank professionals, hematologists, the nursing area, transport, anesthesiologists and intensivists, among others. Consequently, the protocol becomes more widely disseminated outside the specific area, facilitating its application. At morbidity and mortality meetings, this multi-disciplinary approach was maintained in the medical chart review process. Suggestions for enhancing the process were made to the group as a whole and not only the professionals that handled the cases. All the specialties and professionals involved are kept abreast from an educational standpoint.

The Itreg was developed by ECO in conjunction with SBAIT based on the San Diego trauma registry (CA). 
Tailoring to meet Brazilian needs was overseen by a working group from the SBAIT that drew on services with greater experience in data collection in Brazil, with the wider goal of devising a Brazilian Trauma Registry (Brazil TR). The Brazil TR can provide an overview of the characteristics of trauma on a national scale hitherto never seen. The possibility of benchmarking would allow more effective selfassessment, contributing to an improvement in the quality of trauma patient care. In the present study, this software was applied in a pioneering program which, allied with an appropriate quality program, proved highly effective in evidencing the needs for improvements in the service. Moreover, application of the itreg software makes the creation of the Brazil TR feasible.

It is noteworthy that the mere implementation of the quality program has led to clear changes in the registering of information. The fact that professionals are aware there is documentation and observation of events has had an extremely positive effect. The trauma registry and trauma care quality improvement program have proven fundamental and vital tools for hospitals responsible for handling this special patient group.

\title{
RE S U M O
}

\begin{abstract}
Objetivo: analisar a implantação de registro de trauma em hospital universitário com atendimento ao SUS, bem como, sua capacidade em identificar pontos para melhorada qualidade no atendimento. Métodos: o grupo de coleta de dados foi composto por alunos dos cursos de medicina e enfermagem, orientados pelos coordenadores do projeto. Utilizamos o software itreg (ECO Sistemas-RJ/SBAIT) como ferramenta de banco de dados. Vários "filtros" de qualidade foram propostos no intuito de selecionar os casos a serem revistos no processo de controle de qualidade. Resultados: entre março e novembro de 2014, foram inseridos no itreg dados de 1344 vítimas de trauma. Cerca de 87\% foram vítimas de trauma fechado, 59,6\% apresentaram RTS>7,0 e, 67\%, ISS $<9$. Os registros foram completos em 292 casos, que foram selecionados para a revisãono programa de qualidade. Os filtros de auditoria mais frequentemente anotadosforam a laparotomia após quatro horas da admissão e a drenagem dos hematomas subdurais agudos após quatro horas da admissão. Identificamos vários pontos a serem desenvolvidos, como o controle da "supertriagem" de doentes, a necessidade de diminuição do número de exames de imagem negativos, o desenvolvimento de protocolos para passagem de acessos venosos centrais e tratamento de trauma craniencefálico grave. Conclusão: o Registro de Trauma traz uma visão clara dos pontos a serem melhorados no atendimento ao traumatizado, contudo, há características específicas na implantação desta ferramenta.
\end{abstract}

Descritores: Traumatismo Múltiplo. Ferimentos e Lesões. Índices de Gravidade do Trauma. Registros Médicos. Controle de Qualidade.

\section{REFERENCES}

1. Brasil. Ministério da Saúde. Datasus. Sistema de Informação sobre Mortalidade - SIM [Internet]. Brasília; 2013 [acessado em: 10jan2015]. Disponível em: http://tabnet.datasus.gov.br/cgi/ tabcgi.exe?sim/cnv/obt10uf.def

2. Reichenheim ME, de Souza ER, Moraes $C L$, de Mello Jorge $M H$, da Silva CM, de Souza Minayo MC. Violence and injuries in Brazil: the effect, progress made, and challenges ahead. Lancet. 2011;377(9781):1962-75

3. Brasil. Ministério da Saúde. Gabinete do Ministro. Portaria n 1365 08 de julho de 2013. Aprova e institui a linha de cuidado ao trauma na rede de atenção às urgências e emergências. Diário oficial da União 29 ago 2013; Seção 1.

4. Zehtabchi S, Nishijima DK, McKay MP, Mann NC. Trauma registries: history, logistics, limitations, and contributions to emergency medicine research. AcadEmerg Med. 2011;18(6):637-43.

5. Rogers SC, Campbell BT, Saleheen H, Borrup K, Lapidus G. Using trauma registry data to guide injury prevention program activities. J Trauma. 2010;69(4 Suppl):S209-13.

6. Nathens $A B$, Cryer HG, Fildes J. The American College of Surgeons Trauma Quality Improvement Program. SurgClin North Am. 2012:92(2):441-54.

7. Society of Trauma Nurses. Trauma Outcomeand Performance Improvement Course (TOPIC).Acessado em: 10jun2015. Disponível em: http://www.traumanurses.org/topic

8. World Health Organization. Guidelines for trauma quality improvement programmes. Geneve: World Health Organization; 2009
9. American College of Surgeons. Committee on Trauma. Resources for optimal care of the injured patient. $6^{\text {th }}$ ed. Chicago, IL: American College of Surgeons; 2014.

10. Stelfox HT, Bobranska-Artiuch B, Nathens A, Straus SE.Quality indicators for evaluating trauma care: a scoping review. Arch Surg. 2010;145(3):286-95.

11. American College of Surgeons. National Trauma Data Bank. Acessado em: 10jun2015. Disponível em:http://bulletin.facs.org/ 2015/01/annual-report-2014-how-severe-is-it/

12. Teasdale G, Jennett B. Assessment of coma and impaired consciousness. A practical scale.Lancet.1974;2(7872):81-4.

13. Champion HR, Sacco WJ, Copes WS, Gann DS, Gennarelli TA, Flanagan ME. A revision of the Trauma Score. J Trauma. 1989;29(5):623-9.

14. Association for the Advancement of Automotive Medicine (USA), Committee on Injury Scaling. The abbreviated injury scale-1990 Revision (AIS-90). Des Plaines, IL: Association for the Advancement of Automotive Medicine, 1990.

15. Baker SP, O'Neill B, Haddon W Jr, Long WB. The injury severity score: a method for describing patients with multiple injuries and evaluating emergency care. J Trauma. 1974;14(3):187-96.

16. Domingues CA, Sousa RMC, Nogueira LS, Poggetti RS, Fontes $B$, Muñoz D. The role of the New Trauma and Injury Severity Score (NTRISS) for survival prediction. Rev esc enferm USP. 2011;45(6):1353-8

17. Boyd CR, Tolson MA, Copes WS. Evaluating trauma care: the TRISS method. Trauma Score and the Injury Severity Score. Trauma.1987;27(4):370-8. 
18. Cameron PA, Gabbe BJ, McNeil JJ, Finch CF, Smith KL,Cooper DJ, et al. The trauma registry as a statewide quality improvement tool. J Trauma. 2005;59(6):1469-76.

19. Tyson AF, Varela C, Cairns BA, Charles AG. Hospital mortality following trauma: an analysis of a hospital-based injury surveillance registry in sub-Saharan Africa. J Surg Educ. 2015;72(4):e66-72.

20. Juillard CJ, Stevens KA, Monono ME, Mballa GA, Ngamby MK, McGreevy J, et al. Analysis of prospective trauma registry data in Francophone Africa: a pilot study from Cameroon. World J Surg. 2014;38(10):2534-42.

21. Petroze RT, Byiringiro JC, Kyamanywa P, Ntakiyiruta G, Calland JF, Sawyer RG. Infectious outcomes assessment for health system strengthening in low-resource settings: the novel use of a trauma registry in Rwanda. Surg Infect. 2014;15(4):382-6.
22. Alyafei KA, Toaimah F, El Menyar A, Al Thani H, Youssef B, Mollazehi $M$, Consunji R. Analysis of pediatric trauma data from a hospital based trauma registry in Qatar. Int J CritIllnInj Sci. 2015;5(1):21-6.

Received on 08/01/2015

Accepted for publication 23/02/2015

Conflict of interest: none.

Source of funding: FAPESP: Research in Public Policies for the SUS Process:12/51281-7

Address for correspondence:

José Gustavo Parreira

E-mail: jgparreira@uol.com.br 